\title{
Universiteit
}

Leiden

The Netherlands

\section{Informed consent procedures for emergency interventional research in patients with traumatic brain injury and ischaemic stroke}

Kompanje, E.J.O.; Dijck, J.T.J.M. van; Chalos, V.; Berg, S.A. van den; Janssen, P.M.; Nederkoorn, P.J.; ... ; Peul, W.C.

\section{Citation}

Kompanje, E. J. O., Dijck, J. T. J. M. van, Chalos, V., Berg, S. A. van den, Janssen, P. M., Nederkoorn, P. J., ... Peul, W. C. (2020). Informed consent procedures for emergency interventional research in patients with traumatic brain injury and ischaemic stroke. The Lancet Neurology, 19(12), 1033-1042. doi:10.1016/S1474-4422(20)30276-3

Version: $\quad$ Not Applicable (or Unknown)

License: $\quad$ Creative Commons CC BY 4.0 license

Downloaded from: https://hdl.handle.net/1887/3182311

Note: To cite this publication please use the final published version (if applicable). 


\title{
Informed consent procedures for emergency interventional research in patients with traumatic brain injury and ischaemic stroke
}

\author{
Erwin 0 Kompanje*, Jeroen T J M van Dijck*, Vicky Chalos, Sophie A van den Berg, Paula M Janssen, Paul J Nederkoorn, Mathieu van der Jagt, \\ Giuseppe Citerio, Nino Stocchetti, Diederik WJ Dippel, Wilco C Peul
}

\begin{abstract}
Health-care professionals and researchers have a legal and ethical responsibility to inform patients before carrying out diagnostic tests or treatment interventions as part of a clinical study. Interventional research in emergency situations can involve patients with some degree of acute cognitive impairment, as is regularly the case in traumatic brain injury and ischaemic stroke. These patients or their proxies are often unable to provide informed consent within narrow therapeutic time windows. International regulations and national laws are criticised for being inconclusive or restrictive in providing solutions. Currently accepted consent alternatives are deferred consent exception from consent, or waiver of consent. However, these alternatives appear under-utilised despite being ethically permissible, socially acceptable, and regulatorily compliant. We anticipate that, when the requirements for medical urgency are properly balanced with legal and ethical conduct, the increased use of these alternatives has the potential to improve the efficiency and quality of future emergency interventional studies in patients with an inability to provide informed consent.
\end{abstract}

\section{Introduction}

Health-care professionals and researchers have the legal and ethical responsibility to inform patients before executing procedures as part of a clinical study., Each patient has the right to refuse study participation. ${ }^{2}$ This right is internationally recognised and formalised in many declarations, regulations, directives, and laws. ${ }^{1-4}$ For research involving humans, physicians must consider the applicable international norms and standards, as well as their country's general ethical, legal, and regulatory standards. ${ }^{2}$ From a legal perspective, obtaining informed consent is focused on liability and establishing a shared responsibility between professionals and patients, while from a moral perspective, the focus is mostly on respecting autonomous choices and actions of the patient. The process of informed consent is a multidimensional process that serves several important ethical functions. ${ }^{5-7}$

Obtaining informed consent is especially challenging in patients with acute medical emergencies with compromised decision-making capacity from traumatic brain injury and ischaemic stroke because: (1) the short therapeutic time window necessitates urgent intervention without unnecessary delay, (2) the acute or life-threatening condition associated with acute cognitive impairment impedes obtaining valid patient informed consent before intervention, and (3) obtaining consent before intervention from proxies is not always possible, because they cannot always be located or contacted within the time window or they are unable to provide consent for other reasons. These difficulties are probably contributing to the international variation in policy and practice regarding consent procedures for emergency research. ${ }^{8-11}$

Investigating novel, potentially effective therapeutic options for these patients is essential because traumatic brain injury and ischaemic stroke are associated with high rates of mortality and morbidity, which is a major burden for patients, proxies, and societies..$^{12,13}$ Moreover, many available treatments are still largely unproven or of little benefit. $^{12-15}$ To facilitate research to improve health and functional outcome in these patients, several pragmatic solutions are used to overcome the inability of obtaining patient informed consent before urgent medical intervention. However, the legal basis for these solutions is not universally present.

In this Personal View, we outline the theoretical and ethical basis of four different informed consent procedures in emergency interventional research and their use and challenges in common practice, focussing on patients with traumatic brain injury and ischaemic stroke with an inability to provide consent. We also provide procedural recommendations for future emergency research initiatives.

Patient informed consent before medical intervention Patient informed consent before medical intervention is an ethical cornerstone of research involving humans, but obtaining valid patient informed consent before medical intervention for emergency interventional research in traumatic brain injury or ischaemic stroke is challenging. Most patients with severe acute injury from traumatic brain injury or ischaemic stroke have neurological deficits that limit their ability to make or communicate autonomous decisions about research participation. The inability to provide consent is usually caused by a decreased level of consciousness, cognitive impairments, or pharmacological sedation. ${ }^{12,13}$ In patients who are less severely injured, and with variable clinical presentation, this inability can also be difficult to establish. ${ }^{12,13}$ Problems with obtaining consent are frequently caused by factors like cognitive impairment or aphasia. ${ }^{16,17}$ The latter is present in up to $45 \%$ of patients in acute stroke trials, of which $30 \%$ have severe aphasia. ${ }^{18}$ Variability between
Lancet Neurol 2020

Published Online October 21, 2020 https://doi.org/10.1016/ S1474-4422(20)30276-3

See Online/Comment https://doi.org/10.1016/ S1474-4422(20)30367-7 ${ }^{*}$ Contributed equally

Department of Public Health (V Chalos MD), Department of Neurology (V Chalos,

$S A$ van den Berg MD, $P M$ Janssen MD,

D W J Dippel PhD), Department of Radiology and Nuclear Science (V Chalos), Department of Intensive Care Adult (EJ O Kompanje PhD, $M$ van der Jagt $P h D)$, Erasmus Medical Center, University Medical Center, Rotterdam, The Netherlands; Department of Ethics and Philosophy of Medicine, Erasmus University, Rotterdam, The Netherlands (EJ O Kompanje); University Neurosurgical Center Holland, Leiden University Medical Center, Haaglanden Medica Center \& Haga Teaching Hospital, Leiden and The Hague, The Netherlands (JTJ M van Dijck MD, W ( Peul PhD); Department of Neurology, Amsterdam UMC, The Netherlands (S A van den Berg, PJ Nederkoorn PhD); School of Medicine and Surgery, University of Milan-Bicocca, Milan, Italy (G Citerio MD); Department of Physiopathology and Transplantation, Milan University, Milan, Italy (N Stocchetti MD); and Neuro Intensive Care Unit, Fondazione Erasmus Medical Center, Ca Granda Ospedale Maggiore Policlinico Milano, Milan, Italy (N Stocchetti) 


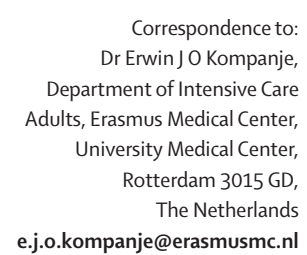

Panel 1: Prerequisites for obtaining valid patient or proxy informed consent before intervention

\section{Disclosure}

The patient or proxy should be provided with complete and understandable information about the purpose, duration, potential risks or benefits, and possible other consequences of the study.

\section{Understanding}

The patient or proxy should fully understand all provided information.

\section{Authenticity}

The patient or proxy can make a judgement, which is consistent with the patient's personal values.

\section{Non-control}

The patient or proxy should be able to make a decision without coercion, manipulation, or other undue influences.

\section{Capacity}

The patient or proxy should be able to oversee the consequences of providing informed consent and thereby study participation

\section{Intentionality}

The patient or proxy should have the intention to participate in the study.

\section{Time}

The patient or proxy should be provided sufficient time to decide on informed consent for study participation.

injuries, and especially injury severity, has implications for how consent might need to be approached. To avert consent problems, researchers have adjusted study protocols by excluding patients with aphasia, left-hemisphere stroke, and moderate or severe cognitive impairment. This approach could, however, cause selection bias and limit external validity of study results. ${ }^{10,19-22}$

Several measures of capacity have been proposed to provide more accurate measurement of decisional capacity, but all have substantial limitations. ${ }^{7,23}$ We propose several conditions that could be used to help determine the validity of patient consent before intervention (panel 1). When determining consent validity, researchers must balance between two undesirable extremes: (1) having a low threshold for inclusion and a risk of including patients who might not understand what they are agreeing to, and (2) having a high threshold for inclusion and including patients without trying to get their consent at all.

In the context of emergency interventional research in traumatic brain injury and ischaemic stroke, time constraints make it impossible to await recovery to provide valid patient consent before intervention. Although consent is often obtained in parallel with imaging, laboratory tests, or readying an angiographic suite or operating theatre, obtaining patient consent before intervention could further delay treatment. This approach is problematic because study interventions might need to be delivered in a very short therapeutic time window to be effective. ${ }^{24,25}$ Secondary brain injury after traumatic brain injury can be less severe when treatment is initiated early ${ }^{26}$ and stroke outcomes are better when reperfusion therapy is administered at the earliest opportunity. ${ }^{27,28}$ A delay of $1 \mathrm{~h}$ in reperfusion time in patients with ischaemic stroke is associated with an increase of absolute risk of $6 \cdot 0-7.7 \%$ for unfavourable functional outcome (modified Rankin Scale score 0-2)..$^{29,30}$ The ULTRA-study ${ }^{31}$ included patients with decisional capacity without patient consent before intervention because delay in ultra-early administration of the study intervention could compromise its potential effect, and thereby invalidate trial design and trial outcome. Obtaining consent was even considered unethical because patients would have been exposed to unnecessary risk. ${ }^{31}$ Several options to minimise time-to-consent have been suggested, ranging from information leaflets to the use of electronic consenting by telemedicine or smartphones. ${ }^{32-34}$ Nonetheless, many studies have described recruitment problems related to informed consent procedures. ${ }^{21,26,35}$ These problems are not limited to patients in acute care settings, but also occur when patients are exposed to continued and prolonged study activities.

To determine the approaches to informed consent procedures used by traumatic brain injury and ischaemic stroke researchers, we examined a representative sample of randomised controlled trials in emergency traumatic brain injury $(\mathrm{n}=70)$ and ischaemic stroke $(\mathrm{N}=76)$ literature (appendix pp 3-16; panel 2). Type of consent was reported in $61(87 \%)$ of 70 randomised controlled trials on traumatic brain injury and in 71 (93\%) of 76 randomised controlled trials on ischaemic stroke. Patient consent before medical intervention was mentioned to be the only consent option in $3(5 \%)$ of 61 randomised controlled trials on traumatic brain injury and five (7\%) of 71 randomised controlled trials on ischaemic stroke. In total, patient consent before intervention was reported to be an option in 15 (25\%) of 61 randomised controlled trials on traumatic brain injury and $68(96 \%)$ of 71 randomised controlled trials on ischaemic stroke (table; panel 3). Obtaining patient consent before intervention was often stated to be impossible because of the sustained brain injury (appendix pp 3 , 16-17). In these cases, researchers resorted to three alternatives to patient informed consent before intervention, proxy informed consent before intervention, deferred consent, and exception from informed consent or waiver of consent.

\section{Proxy informed consent before intervention}

Proxy informed consent before intervention was the most commonly used alternative for patient consent before intervention and used in most randomised controlled trials on traumatic brain injury (56 [92\%] of 61) and ischaemic stroke (63 [89\%] of 71; table). Proxy informed consent before intervention is provided by an individual 
who has the legal right to provide consent on behalf of the patient. There are many descriptions in the literature because the legal base that regulates the selection of individuals to act as proxy is variable: consent by a family member, a relative, an appointed person or legally authorised representative; surrogate or substitute decision maker; guardian permission; and sometimes independent physician consent. Independent physicians could serve as proxies for informed consent decisions in two (3\%) of 61 randomised controlled trials on traumatic brain injury and in two (3\%) of 71 trials on ischaemic stroke. The conditions listed in panel 1 could also be considered to assess validity of proxy informed consent before intervention. Examples of where proxy informed consent before intervention is approved include the Australia, Ethiopia, European Union, Chile, China, India, Japan, North America, South Africa, and New Zealand, and is described as valid in the Declaration of Helsinki ${ }^{2}$ and the International Ethical Guidelines for Health-related Research Involving Humans (appendix p 21). ${ }^{4}$

The two main barriers to obtain proxy informed consent before intervention in emergency research are the short therapeutic time window that precludes a consent conversation, and the fact that proxies cannot always be located or contacted. ${ }^{21,35,36}$ As with patient consent before intervention, delaying a timely start of study interventions to obtain proxy informed consent before intervention is undesirable as it can decrease the efficacy of the acute therapy. ${ }^{26-28}$

A third barrier is that proxy decision making in research is highly complex and, although proxies prefer to be involved, empirical evidence suggests that proxies might not always be suitable as surrogate decision makers. ${ }^{37,38}$ Substantial discrepancies are described between decisions of patients and proxies in hypothetical scenarios. ${ }^{39,40}$ About $50 \%$ of proxies reported to be comfortable with being involved, but many are also emotionally overwhelmed, stressed, distracted, or report symptoms of anxiety and depression. ${ }^{37,41-43}$

Proxies aim to make a decision that is authentic to the person they represent by balancing factors such as patients values, preferences, and wellbeing. ${ }^{38,44-47}$ Other factors that affected decisions include the time sensitivity of the decision, perceived study risk or benefit, uncertainty of possible outcomes, the complexity of the patient's condition, the use of medical terminology, and communication with physicians and nurses. ${ }^{37,47,48}$ Study participation is often declined because proxies feel unable or unwilling to consider it. ${ }^{49,50}$ Other common reasons to decline consent were being too anxious (67\%), fear of experimental treatment (37\%), and concerns about risks (33\%). ${ }^{44}$ Reasons to provide consent were wanting to help others (91\%), contributing to medical progress $(88 \%)$, and trusting (87\%) or not wanting to disappoint the medical team $(10 \%) .^{44}$

In summary, alternatives to patient or proxy informed consent before intervention are sometimes needed in traumatic brain injury or ischaemic stroke emergency
Panel 2: Search strategy and selection criteria

We searched PubMed and Ovid MEDLINE using several strategies. To be informed about the used consent procedures in current traumatic brain injury and ischaemic stroke emergency research practice, we used a representative selection of randomised controlled trials. Data on study design and used consent procedures were extracted. Details on the search strategies, article selection procedures, data extraction, and synthesis of results can be found in the appendix $\mathrm{p} 3-17$. We found articles on the theoretical and conceptual aspects of consent procedures specifically for patients with traumatic brain injury and stroke using search terms, including 'informed consent', 'brain injuries', 'head injuries', and 'stroke' (appendix p 18). We focussed on theoretical and conceptual articles about the most commonly used consent procedures (appendix $\mathrm{p} 20$ ). This search strategy formed the evidence base for this Personal View.

\begin{tabular}{|c|c|c|}
\hline & $\begin{array}{l}\text { Traumatic brain } \\
\text { injury }(\mathrm{N}=70)\end{array}$ & $\begin{array}{l}\text { Ischaemic stroke } \\
(\mathrm{N}=76)\end{array}$ \\
\hline Type of consent reported & $61(87 \%)$ & $71(93 \%)$ \\
\hline $\begin{array}{l}\text { Patient informed consent before } \\
\text { medical intervention }\end{array}$ & $15(25 \%)$ & $68(96 \%)$ \\
\hline $\begin{array}{l}\text { Proxy informed consent before } \\
\text { intervention }\end{array}$ & $56(92 \%)$ & $63(89 \%)$ \\
\hline Deferred consent & $8(13 \%)$ & $3(4 \%)$ \\
\hline $\begin{array}{l}\text { Exception from informed consent } \\
\text { waiver of informed consent }\end{array}$ & $6(10 \%)$ & $5(7 \%)$ \\
\hline $\begin{array}{l}\text { Physician consent or other } \\
\text { consent type }\end{array}$ & $2(3 \%)$ & $2(3 \%)$ \\
\hline \multicolumn{3}{|l|}{ Data are $n(\%)$} \\
\hline
\end{tabular}

interventional research because of the short therapeutic time windows, the deficits caused by traumatic brain injury or ischaemic stroke, and the frequent lack of available proxies. All factors preclude determining a patient's preferences. When patient or proxy informed consent before intervention are not practicable, the use of consent alternatives is imperative. ${ }^{51}$

\section{Deferred consent}

This procedure allows participants to be included in studies when patients and proxies are unable to provide valid previous consent within short time frames. The approach was infrequently reported as an option in our analysed sample of randomised controlled trials on traumatic brain injury (eight [13\%] of 61) and ischaemic stroke (three [4\%] of 71), nearly always in addition to patient and proxy informed consent before intervention (table). It is usually described as deferred patient or proxy consent, retrospective consent, delayed consent, implied consent and consent to continue, or reconsent from patient, and is 
Panel 3: Comparison of consent procedures in traumatic brain injury and ischaemic stroke literature

There are similarities and differences between the types of consent reported in traumatic brain injury and ischaemic stroke literature (appendix p 16). First, the patient consent before intervention option was reported to be used less frequently in randomised controlled trials on traumatic brain injury (25\%) than in randomised controlled trials on ischaemic stroke (96\%; table). This difference does not necessarily mean that patient consent before intervention was impermissible when a participants' consent capacity was intact, but could also mean that it was not considered applicable or relevant for the study population. The difference likely depends on patient and study characteristics and is probably related to a perceived continued ability to provide patient informed consent before intervention after ischaemic stroke in most patients, whereas traumatic brain injury generally has a greater effect on this ability. This might be especially true in the case of more severe traumatic brain injury, additional extracranial injury, and a need for intensive care unit admission.

Second, the reported possibility to use proxy informed consent before intervention was very high in both literature on traumatic brain injury (92\%) and ischaemic stroke (88\%), and the use of independent physician consent procedures was equally low (3.3\% vs $2.8 \%$ ).

Third, the use of deferred consent and exception from consent was higher in randomised controlled trials on traumatic brain injury (23\%) than in those on ischaemic stroke (11\%), probably for the same reasons as reported for patient informed consent before intervention differences. There seems to be an increase in randomised controlled trials allowing patient recruitment without patient informed consent before intervention or proxy informed consent before intervention; however, many studies did not use it as an alternative for patient informed consent or proxy informed consent before intervention.

Last, there were more missing descriptions of consent procedures in the literature on traumatic brain injury (13\%) than on ischaemic stroke (6.6\%), which is likely caused by the inclusion of more dated randomised controlled trials on traumatic brain injury. Nearly all newer studies included a description of informed consent procedures.

allowed and practised in places such as the Australia, European Union, China, India, Japan, and South Africa. It is described as valid in the Declaration of Helsinki ${ }^{2}$ and in the International Ethical Guidelines for Health-related Research Involving Humans. ${ }^{4}$ After starting study procedures without patient informed consent before intervention or proxy informed consent before intervention, consent must be obtained for study continuation as soon as patients or proxies regain the ability to provide consent. Some authors recommend a time limit of $72 \mathrm{~h}$ to prevent unauthorised use of conducting research without previous consent, ${ }^{41}$ but there is no legal or moral ground for this recommendation..$^{52}$ When it remains impossible to get affirmative consent for study continuation for reasons other than death, it could be necessary to withdraw patients from the study. This depends on the specific study circumstances and procedures as reviewed and approved by a responsible institutional review board. When consent for study continuation is provided, already collected data can be used. When study continuation is refused, already collected data can still be used when patients or proxies do not use their right to refuse this.

The procedural particulars depend on local legislation, institutional review board requirements, and their assessment of the relative pros and cons. Respecting local requirements is important, but also has a risk of practice variation and use of different terms or descriptions, both resulting in indistinctness, misunderstanding, and even misuse. ${ }^{8-10}$ Researchers should be aware of this possibility and multinational studies therefore need to be flexible enough to tailor their approach to all applicable requirements. ${ }^{53}$ Although most researchers use the deferred consent procedure to obtain consent for study continuation, it is sometimes interpreted as a requirement to obtain consent for research activities that have already taken place. However, considering the earlier suggested conditions (panel 1) and the actual meaning of consent (give permission for something to happen or agreement to do something), it can only be concluded that asking and obtaining valid consent is possible only for research activities in the future.

Many patients and proxies report to be willing to participate in a study without previous consent. ${ }^{42,50,54,55}$ Although the deferred consent procedure was not always supported afterwards, ${ }^{56}$ most proxies of patients included in acute care studies (81-100\%) without previous informed consent agreed to further participation..$^{49,56-58}$ Only few patients that refused further participation also denied permission for the use of already collected data. ${ }^{58}$ Experienced stress in the setting of an intensive care unit admission was commonly mentioned as reason to endorse the use of a deferred consent procedure. ${ }^{42}$

A deferred consent procedure is also being used in three ongoing randomised controlled trials on modifications of endovascular treatment for acute ischaemic stroke (MR CLEAN-MED, MR CLEAN-NO IV, MR CLEAN LATE) within the CONTRAST consortium..$^{59}$ On Nov 8, 2019, preliminary data were available for 742 patients of these CONTRAST studies, of whom 664 (90\%) patients or proxies provided written consent after the trial treatment, and $36(5 \%)$ patients died before consent could be obtained. Written consent for study continuation was not obtained in 42 patients (6\%), of whom half did not object to the use of already collected data. The observation, that postponing consent until after the study treatment is usually accepted by patients and proxies, has been shown in previous (non-stroke) clinical studies..$^{43,55}$

In the CONTRAST studies, the median time from admission at the intervention centre to randomisation was 25 min (IQR 16-39), which was shorter than the earlier MR CLEAN trial (76 min; IQR 48-144). ${ }^{60}$ In the MR CLEAN trial, ${ }^{60}$ which compared endovascular treatment with usual care versus usual care alone, written patient or proxy informed consent before intervention was obtained based on oral communication and an abbreviated information letter. ${ }^{60}$ Written consent was asked again after the acute phase. Although workflow has improved substantially over time, the difference between these time intervals could suggest that valuable time is lost when using patient consent or proxy informed consent before intervention. This additional time can delay intervention, which could negatively affect effectiveness of the acute intervention. ${ }^{29,30}$ 
Emergency research in acute traumatic brain injury and ischaemic stroke often includes patients who die after being included without patient or proxy informed consent before intervention. Exclusion of included patients who have died before consent was obtained is obviously undesirable, as it reduces statistical power, introduces selection bias, causes asymmetrical randomisation, and decreases external validity. ${ }^{41,61}$ When privacy is guaranteed, using already collected data is judged to be ethically valid.41,61 Explicit proxy consent is not required in these circumstances. Retrospective removal of study patients from a database, after randomisation, for any reason, not just death, is even considered to be a threat to the scientific integrity of the trial. Scientific integrity is necessary for any trial to be ethically justifiable.

\section{Exception from consent}

Exception from consent was used in six (10\%) of 61 randomised controlled trials on traumatic brain injury and in five $(7 \%)$ of 71 on ischaemic stroke and is also called waiver of informed consent. By contrast to the deferred consent procedure, patient or proxy informed consent are not required for continuation of study-related activities if the patient or a proxy never becomes available to engage in an informed consent process, despite diligent goodfaith efforts by the researchers. It is particularly practiced in North America and Ethiopia, and described as valid in the Declaration of Helsinki ${ }^{2}$ and in the International Ethical Guidelines for Health-related Research Involving Humans. ${ }^{4}$ In an effort to improve the progress in emergency research involving patients unable to provide informed consent, the US American Food and Drugs Administration (FDA) published guidelines in 1996, describing the exception from informed consent requirements for emergency research and the waiver of informed consent (appendix p 21). Since the guidelines, exception from informed consent has been available for use in emergency research for US FDA regulated products and waiver of informed consent for non-FDA regulated products.

With this alternative, a study can start without patient or proxy informed consent before intervention. Relevant information on study participation and use of data should be communicated to patients or proxies at the earliest opportunity. Refusal of study continuation or use of already obtained data should always be respected. The exception from informed consent procedure could be necessary when patients are exposed to continued and prolonged study activities while obtaining patient or proxy informed consent before intervention is not possible. The participant remains in the study by default.

Community consultation or public disclosure are specifically required to support the use of exception from informed consent or waiver of informed consent and aim to protect the rights and welfare of study participants. ${ }^{62}$ In community consultation, representatives from general communities (geographic community) or from the population at risk for the condition (condition-oriented community) are recruited. It aims to involve and engage community members with research initiatives by using public fora, community groups, or face-to-face and telephone surveys. Public disclosure involves notifying the community in advance that patients will be enrolled in a study in an emergency situation without patient or proxy informed consent before intervention. After the study, results will be communicated to participants and the public. It remains unclear whether patients, proxies, health-care providers, administrators, or a general population should be considered to be the community. ${ }^{63}$ Although some reports are positive and participants satisfied, ${ }^{64,65}$ community consultation and public disclosure are also challenging, time consuming, and costly. ${ }^{66,67}$

A study ${ }^{68}$ reviewed 28 completed and published acute care studies between 1996 and 2018, that used exception from informed consent or waiver of informed consent. ${ }^{68}$ Only $359(0.6 \%)$ of 63947 study enrolments were withdrawn or did not provide consent for continued study participation. ${ }^{68}$ Acceptance of the exception from informed consent procedure was high and varied by the specifics of the situation. ${ }^{65,69}$

\section{Implications for research practice}

The difficulties regarding patient and proxy informed consent before study intervention in traumatic brain injury and ischaemic stroke emergency interventional research can result in many lost research opportunities when alternatives for informed consent are not facilitated. ${ }^{43}$ Based on the sample of randomised controlled trials, patient recruitment without patient and proxy informed consent before study intervention seems to be increasingly used in recent years, but still many studies do not use it. The use and efficiency of consent procedures in traumatic brain injury and ischaemic stroke emergency research should be improved.

Selecting an appropriate informed consent procedure for a study is difficult and depends on many factors, often related to each other. Factors include local legislation, institutional review board requirements, and study details such as methods, interventions, and patient characteristics. We propose use of a flow chart to guide investigators or regulators to select the most appropriate informed consent procedures based on several study particulars (figure). Informed consent procedures should be used as overlapping and complementary strategies to solve different challenges of a study. Researchers should first determine whether the therapeutic time window allows time for an informed consent procedure. If there is time, it should also be determined whether it is feasible to obtain valid patient or proxy informed consent before intervention within the time window. The conditions suggested in panel 1 could be used as a starting point to assess consent validity. If both are not practicable, the determination of a patient's wishes regarding study participation should be considered not possible. Researchers should then consider 


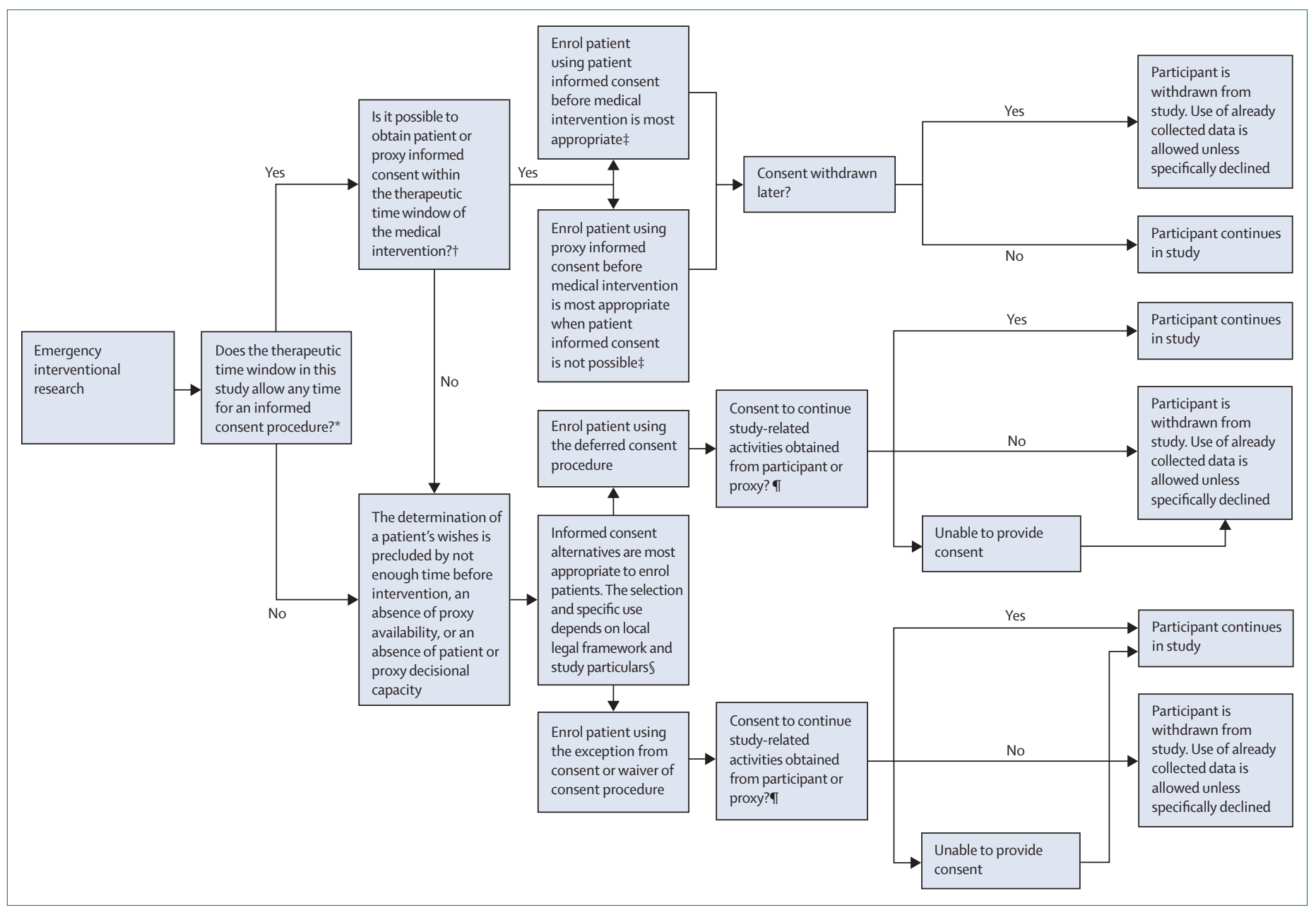

Figure: Flow chart for the selection of appropriate informed consent procedures in emergency interventional research

Proposed flowchart to guide investigators or regulators to select the most appropriate informed consent procedure based on several traumatic brain injury or ischaemic stroke study particulars. Studies could use multiple informed consent procedures in their informed consent strategy. Informed consent procedures should be used as overlapping and complementary strategies to solve different challenges of a study. This flowchart could be best seen as a legal and ethical framework that could be considered in any research setting. It is not binding, and a chosen informed consent strategy should always follow applicable legislation and must be evaluated and approved by the responsible institutional review boards. *Some emergency interventional studies on traumatic brain injury and ischaemic stroke use a very narrow therapeutic time window (ie, mins) that does not allow any time for an informed consent procedure. Obtaining patient or proxy informed consent before intervention is not possible in these situations, because the intervention is immediate. Obtaining informed consent can delay the study intervention. In some studies, any delay of study intervention is problematic because it could compromise the potential effect of the experimental treatment, making the fair interpretation of results difficult. Obtaining informed consent and delaying the study intervention could also be considered unethical because patients would be exposed to unnecessary risk. In other studies, where the therapeutic time window of traumatic brain injury or ischaemic stroke intervention is wider (ie, several hrs), there might be an opportunity to obtain patient or proxy informed consent. †There are many reasons why it could not be possible to obtain patient or proxy informed consent before intervention even when this could have been possible within the therapeutic time window. Reasons include an absence of available proxies, and a patient's or proxy's inability to provide informed consent. ‡Strategies to optimise and support patient or proxy decision making could help to optimise informed consent procedures. SThe use of deferred consent or exception from consent or waiver of consent procedures depends on study particulars and local legal frameworks, including requirements from institutional review boards. Details can be found in the main text of the manuscript and cited references. Their use should be carefully considered and evaluated by researchers and institutional review boards. FInformed consent to continue study-related activities should be obtained as soon as the patient or a proxy can provide informed consent. In case a proxy provided informed consent first, informed consent should be verified with the patient when this becomes possible.

the option of using an alternative procedure like deferred consent or exception from informed consent or waiver of informed consent. This choice mainly depends on local legislation and study details. A non-exclusive list of prerequisites of both procedures, based on existing legislation, as listed in appendix p 21, can be found in panel 4. These prerequisites are not intended to be conclusive, but could assist researchers in determining the appropriateness of the procedure. All procedural decisions should adhere to applicable legislation.
The use of deferred consent or exception from informed consent or waiver of informed consent procedures seems necessary and acceptable in traumatic brain injury and ischaemic stroke emergency interventional research. The seriousness of the potential threats to the welfare and protection of study participants, the scientific integrity of a trial, and public trust in research should however never be underestimated. ${ }^{70,71}$ Independent institutional review boards or steering committees are charged with the protection of patients, researchers, and the public as a whole, by 
Panel 4: A list of prerequisites for the use of deferred consent and exception from consent procedures

General prerequisites for the use of deferred consent and exception from consent procedures

1 The patient has an acute life-threatening situation or an acute medical condition that necessitates urgent (study) procedures because delayed treatment can negatively affect intervention effectiveness or patient outcome. Due to the urgency of the situation, the patient or proxies are unable to provide valid informed consent before intervention.

2 The medical condition causes an inability to provide informed consent before intervention by patient or proxy.

3 There is scientific information that supports the potential for the study treatment to provide a direct benefit to the patient. Available standard treatments are unproven (the scarcity of high-quality evidence that the treatment is effective) or unsatisfactory (the treatment is unsatisfactory due to safety or efficacy issues that require investigation).

4 The risks and burden of study participation are considered acceptable compared with standard treatment, given the potential direct benefit of the study treatment.

5 Researchers or physicians are unaware of any objections for study participation (eg, a written advanced directive).

6 It is reasonably impossible to prospectively identify individuals that are likely to become eligible for study participation in the future, in such a way that patient or proxy informed consent before intervention could be obtained.

7 It is practically impossible to undertake the emergency research when patient or proxy informed consent before intervention is required to start study-related activities.

8 A comprehensive disclosure of study information and study participation to patients and proxies is required at the earliest possible (practicable) opportunity.

9 If the patient dies during the study before informed consent has been obtained, the already collected data can be used according to the study protocol, without the need for proxy informed consent. Proxies should be informed about study participation at the earliest possible (practicable) opportunity.
10 The use of this alternative for patient or proxy informed consent before intervention is accepted by local legislation. Institutional review boards have reviewed and approved the study protocol to prevent misconduct and ascertain patient safety.

\section{Specific prerequisites for deferred consent}

1 It is considered possible to continue essential study-related activities, such as additional interventions or follow-up, when patient or proxy informed consent is required to continue study-related activities. For example, patients or proxies are not expected to have a prolonged inability to provide valid informed consent.

2 Patient or proxy informed consent is required for continuation of study-related activities and should be obtained from the patient or proxy at the earliest possible (practicable) opportunity after regaining the ability to provide informed consent. When study continuation is refused, the patient or proxy has the right to refuse the use of already obtained data.

3 There are no pre-study requirements such as community consultation or public disclosure.

Specific prerequisites for exception from consent

1 It is practically impossible to continue essential studyrelated activities, such as additional interventions or followup, when patient or proxy informed consent is required to continue study-related activities. For example, patients or proxies are expected to have a prolonged inability to provide valid informed consent.

2 Written patient or proxy informed consent is not required for continuation of study-related activities if the patient or a proxy never becomes available to engage in an informed consent process despite diligent good-faith efforts by the researchers. Patients or proxies should be informed about their right to refuse the use of obtained data.

3 To increase acceptance of the proposed study protocol, pre-study requirements such as community consultation or public disclosure could be required. balancing and judging their interests. Several safeguards are used in the process: a rigorous evaluation of study protocols, oversight in study procedures such as patient screening, recruitment, consent procedure, and independent safety monitoring. ${ }^{7}$ Other safeguards could consist of including and consulting more representatives of patients on institutional review boards to weigh in on the ethics of different trial approaches in patients where patient or proxy informed consent before intervention is not possible.

\section{Conclusions and future directions}

There is an urgent need to investigate novel therapeutic options that are potentially effective for patients with traumatic brain injury and ischaemic stroke. A thorough consideration of the multidimensional process of informed consent is required to increase the feasibility and quality of future emergency research initiatives. Researchers should be aware of the international legal and ethical conditions and possibilities. Implementing this knowledge could improve study protocol and procedures.

Supported by an extensive literature base, we conclude that obtaining patient or proxy informed consent before intervention is often not possible in emergency interventional research in patients with traumatic brain injury or ischaemic stroke. This impossibility is primarily caused by the importance of very narrow therapeutic windows, the inability to provide informed consent, or the frequent absence of surrogate decision makers.

Generally accepted alternatives, such as deferred consent and exception from informed consent or waiver of 
informed consent, appear underutilised in traumatic brain injury and ischaemic stroke emergency interventional research, despite being ethically permissible, socially acceptable, and regulatorily compliant. Not being able to use these alternatives complicates emergency interventional research in these patients. Being able to use them, when appropriate, has the potential to optimally test interventions earlier in a patient's course when they are most likely to be effective. If done properly, it also creates an opportunity for more generalisable and equitable clinical trial participation and results. Using these alternatives appears consistent with the desires of most patients most of the time.

Institutional review boards have an important role to prevent misconduct and protect patient safety by reviewing and approving study protocols. Study procedures should be overseen during the study. Researchers should aim to optimise the use of overlapping and complementary informed consent strategies based on the particular circumstances of a study, especially the requirements and constraints on obtaining patient or proxy informed consent before intervention. Harmonisation of laws and regulations between countries should be pursued, while respecting national sovereignty and local cultural preferences. All measures will further improve the efficiency and quality of emergency research initiatives involving patients with an inability to provide informed consent before medical intervention, regardless of disease.

\section{Contributors}

EJOK and JTJMvD contributed equally to this manuscript. EJOK,

JTJMvD, and WCP made substantial contribution to the initial conception of the work. All other authors contributed to the final design of the manuscript. EJOK, JTJMvD, VC, SAvdB, and DWJD contributed to the acquisition of data through conducting a literature review and analysis of original study data. All listed authors made substantial contributions in the interpretation of these data. EJOK, JTJMvD, VC, and WCP were involved in drafting the manuscript and all listed authors critically revised the work for important intellectual content. All listed authors approved the final version of the manuscript to be published. All listed authors agree to be accountable for all aspects of the manuscript and ensure that questions related to the accuracy or integrity of any part of the work are appropriately investigated and resolved.

\section{Declaration of interests}

DWJD reports that Erasmus MC received research grants from Dutch Heart Foundation, Dutch Brain Foundation, AngioCare BV, Covidien, EV3, Medac, Lamepro, Penumbra, Stryker, and Top Medical, Concentric, The Netherlands Organisation for Health Research and Development, Health Holland Top Sector Life Sciences \& Health, Stryker European Operations BV, Thrombolytic Science, LLC, and Cerenovus. GC reports being a member on the Speaker's Bureau and Advisory board of Integra and Neuroptics; receiving grants and personal fees from Integra and Neuroptics; and personal fees from Nestlé and Union Chimique Belge Pharma, all outside of the submitted work. WP reports to be part of the management team of the CENTER-TBI study (NCT02210221) and to be the principal investigator of the Net-QuRe (NL 5761), Ciao@TBI, RESET-ASDH, and the SPARTA trials. All other authors declare no competing interests.

\section{Acknowledgments}

We thank The European Union seventh Framework Program for funding the Collaborative European NeuroTrauma Effectiveness Research in Traumatic Brain Injury (CENTER-TBI), and the Hersenstichting Nederland (Dutch Brain Foundation) for funding the Neurotraumatology Quality Registry (Net-QuRe). We also thank the funders of the CONTRAST consortium: Netherlands Cardiovascular Research Initiative, an initiative of the Dutch Heart Foundation, the Brain Foundation Netherlands, Health Holland, Top Sector Life Sciences, Medtronic, and Cerenovus. Funders had no involvement in the study design, the collection, analysis and interpretation of data, the writing of this personal view, and the decision to submit the paper for publication.

\section{References}

1 Grady C, Cummings SR, Rowbotham MC, McConnell MV, Ashley EA, Kang G. Informed Consent. N Engl J Med 2017; 376: 856-67.

2 World Medical Association. Declaration of Helsinki. Ethical principles for medical research involving human subjects. 2018 2019. https://www.wma.net/policies-post/wma-declaration-ofhelsinki-ethical-principles-for-medical-research-involving-humansubjects/2194 (accessed Dec 19, 2019).

3 Wolf SM, Clayton EW, Lawrenz F. The past, present, and future of informed consent in research and translational medicine. J Law, Med Ethics 2018; 46: 7-11.

4 Council for International Organizations of Medical Sciences in collaboration with the World Health Organization. International ethical guidelines for health-related research involving humans, fourth edition. 2016. https://cioms.ch/wp-content/uploads/2017/01/ WEB-CIOMS-EthicalGuidelines.pdf (accessed Dec 19, 2019).

5 Dickert NW, Eyal N, Goldkind SF, et al. Reframing consent for clinical research: a function-based approach. Am J Bioeth 2017; 17: 3-11.

6 Bruni T, Graham M, Norton L, Gofton T, Owen AM, Weijer C. Informed consent for functional MRI research on comatose patients following severe brain injury: balancing the social benefits of research against patient autonomy. J Med Ethics 2019; 45: 299-303.

7 Johnson-Greene D. Informed consent issues in traumatic brain injury research: current status of capacity assessment and recommendations for safeguards. J Head Trauma Rehabil 2010; 25: $145-50$.

8 Kompanje EJ, Maas AI, Menon DK, Kesecioglu J. Medical research in emergency research in the European Union member states: tensions between theory and practice. Intensive Care Med 2014; 40: 496-503.

9 van Belle G, Mentzelopoulos SD, Aufderheide T, May S, Nichol G. International variation in policies and practices related to informed consent in acute cardiovascular research: results from a 44 country survey. Resuscitation 2015; 91: 76-83.

10 Majersik JJ. Ethics and bias in clinical trial enrollment in stroke. Curr Cardiol Rep 2019; 21: 49.

11 van Wijk RPJ, van Dijck JTJM, Timmers M, et al. Informed consen procedures in patients with an acute inability to provide informed consent: Policy and practice in the CENTER-TBI study. J Crit Care 2020; 59: 6-15

12 Maas AIR, Menon DK, Adelson PD, et al. Traumatic brain injury: integrated approaches to improve prevention, clinical care, and research. Lancet Neurol 2017; 16: 987-1048.

13 Campbell BCV, De Silva DA, Macleod MR, et al. Ischaemic stroke. Nat Rev Dis Prim 2019; 5: 70.

14 Powers WJ, Rabinstein AA, Ackerson T, et al. Guidelines for the early management of patients with acute ischemic stroke: 2019 update to the 2018 guidelines for the early management of acute ischemic stroke: a guideline for healthcare professionals from the American Heart Association/American Stroke Association. Stroke 2019; 50: e344-418.

15 Carney N, Totten AM, O’Reilly C, et al. Guidelines for the management of severe traumatic brain injury, fourth edition. Neurosurgery 2017; 80: 6-15.

16 Mendyk AM, Labreuche J, Henon $\mathrm{H}$, et al. Which factors influence the resort to surrogate consent in stroke trials, and what are the patient outcomes in this context? BMC Med Ethics 2015; 16: 26.

17 Janssen PM, Chalos V, van den Berg SA, et al. Neurological deficits in stroke patients that may impede the capacity to provide informed consent for endovascular treatment trials. J Stroke Cerebrovasc Dis 2019; 28: 104447.

18 Ali M, Lyden P, Brady M. Aphasia and dysarthria in acute stroke: recovery and functional outcome. Int J Stroke 2015; 10: 400-06.

19 Hotter B, Ulm L, Hoffmann S, et al. Selection bias in clinical stroke trials depending on ability to consent. BMC Neurol 2017; 17: 206. 
20 Thomalla G, Boutitie F, Fiebach JB, et al. Effect of informed consent on patient characteristics in a stroke thrombolysis trial. Neurology 2017; 89: 1400-07.

21 Stanley RM, Johnson MD, Vance C, et al. Challenges enrolling children into traumatic brain injury trials: an observational study. Acad Emerg Med 2017; 24: 31-39.

22 Mendelson SJ, Aggarwal NT, Richards C, O’Neill K, Holl JL, Prabhakaran S. Racial disparities in refusal of stroke thrombolysis in Chicago. Neurology 2018; 90: e359-64.

23 Feng KL, Person C, Phillips-Sabol J, et al. Comparison between a standardized questionnaire and expert clinicians for capacity assessment in stroke clinical trials. Stroke 2014; 45: e229-32.

24 Gomez CR. Time is brain: the stroke theory of relativity J Stroke Cerebrovasc Dis 2018; 27: 2214-27.

25 Zinkstok SM, Beenen LF, Luitse JS, Majoie CB, Nederkoorn PJ Roos YB. Thrombolysis in stroke within 30 minutes: results of the acute brain care intervention study. PLoS One 2016; 11: e0166668.

26 Mohamadpour M, Whitney K, Bergold PJ. The importance of therapeutic time window in the treatment of traumatic brain injury. Front Neurosci 2019; 13: 7 .

27 Saver JL, Goyal M, van der Lugt A, et al. Time to treatment with endovascular thrombectomy and outcomes from ischemic stroke: a meta-analysis. JAMA 2016; 316: 1279-89.

28 Emberson J, Lees KR, Lyden P, et al. Effect of treatment delay, age, and stroke severity on the effects of intravenous thrombolysis with alteplase for acute ischaemic stroke: a meta-analysis of individual patient data from randomised trials. Lancet 2014 384: 1929-35.

29 Fransen PSS, Berkhemer OA, Lingsma HF, et al. Time to reperfusion and treatment effect for acute ischemic stroke: a randomized clinical trial. JAMA Neurol 2016; 73: 190-96.

30 Mulder MJHL, Jansen IGH, Goldhoorn R-JB, et al. Time to endovascular treatment and outcome in acute ischemic stroke: MR CLEAN registry results. Circulation 2018; 138: 232-40.

31 Post R, Germans MR, Coert BA, Rinkel GJE, Vandertop WP, Verbaan D. Update of the ULtra-early TRranexamic Acid after Subarachnoid Hemorrhage (ULTRA) trial: statistical analysis plan. Trials 2020; 21: 199.

32 Haussen DC, Craft L, Doppelheuer S, et al. Legal authorized representative experience with smartphone-based electronic informed consent in an acute stroke trial. J Neurointerv Surg 2020; 12: $483-85$

33 Shoirah H, Wechsler LR, Jovin TG, Jadhav AP. Acute stroke trial enrollment through a telemedicine network: a 12-year experience. J Stroke Cerebrovasc Dis 2019; 28: 1926-29.

34 Berge E, Stapf C, Al-Shahi Salman R, et al. Methods to improve patient recruitment and retention in stroke trials. Int J Stroke 2016; 11: $663-76$

35 Kim D-H, Saver JL, Starkman S, et al. Enrollment yield and reasons for screen failure in a large prehospital stroke trial. Stroke 2016; 47: 232-5.

36 Burns KE, Zubrinich C, Tan W, et al. Research recruitment practices and critically ill patients. A multicenter, cross-sectional study (the Consent Study). Am J Respir Crit Care Med 2013; 187: 1212-18.

37 Barrett KA, Ferguson ND, Athaide V, et al. Surrogate decision makers' attitudes towards research decision making for critically ill patients. Intensive Care Med 2012; 38: 1616-23.

38 Shepherd V, Hood K, Sheehan M, Griffith R, Wood F. 'It's a tough decision': a qualitative study of proxy decision-making for research involving adults who lack capacity to consent in UK. Age Ageing 2019; 48: 903-09.

39 Bryant J, Skolarus LE, Smith B, Adelman EE, Meurer WJ. The accuracy of surrogate decision makers: informed consent in hypothetical acute stroke scenarios. BMC Emerg Med 2013; 13: 18.

40 Newman JT, Smart A, Reese TR, Williams A, Moss M. Surrogate and patient discrepancy regarding consent for critical care research. Crit Care Med 2012; 40: 2590-94.

41 Jansen TC, Kompanje EJO, Druml C, Menon DK, Wiedermann CJ, Bakker J. Deferred consent in emergency intensive care research: what if the patient dies early? Use the data or not? Intensive Care Med 2007; 33: 894-900.

42 Terry MA, Freedberg DE, Morris MC. An alternative consent process for minimal risk research in the ICU. Crit Care Med 2017; 45: $1450-6$.
43 Ecarnot F, Quenot JP, Besch G, Piton G. Ethical challenges involved in obtaining consent for research from patients hospitalized in the intensive care unit. Ann Transl Med 2017; 5: s41.

44 Mehta S, Quittnat Pelletier F, Brown M, et al. Why substitute decision makers provide or decline consent for ICU research studies: a questionnaire study. Intensive Care Med 2012; 38: 47-54.

45 Hwang DY, Knies AK, Mampre D, et al. Concerns of surrogate decision makers for patients with acute brain injury: a US population survey. Neurology 2020; 94: e2054-68.

46 Devnani R, Slaven JEJ, Bosslet GT, et al. How surrogates decide: a secondary data analysis of decision-making principles used by the surrogates of hospitalized older adults. J Gen Intern Med 2017; 32: 1285-93.

47 Burns KE, Prats CJ, Maione M, et al. The experience of surrogate decision makers on being approached for consent for patient participation in research. A multicenter study. Ann Am Thorac Soc 2017; 14: 238-45.

48 Long B, Clark L, Cook P. Surrogate decision making for patients with severe traumatic brain injury. I Trauma Nurs 2011; 18: 204-12.

49 Topolovec-Vranic J, Santos M, Baker AJ, Smith OM, Burns KE. Deferred consent in a minimal-risk study involving critically ill subarachnoid hemorrhage patients. Can Respir J 2014; 21: 293-96.

50 Woolfall K, Frith L, Gamble C, Gilbert R, Mok Q, Young B. How parents and practitioners experience research without prior consent (deferred consent) for emergency research involving children with life threatening conditions: a mixed method study. BMJ Open 2015; 5: e008522.

51 Rebers S, Aaronson NK, van Leeuwen FE, Schmidt MK. Exceptions to the rule of informed consent for research with an intervention. BMC Med Ethics 2016; 17: 9.

52 Jansen TC, Kompanje EJ, Bakker J. Deferred proxy consent in emergency critical care research: ethically valid and practically feasible. Crit Care Med 2009; 37: S65-68.

53 CRASH-3 trial collaborators. Effects of tranexamic acid on death, disability, vascular occlusive events and other morbidities in patients with acute traumatic brain injury (CRASH-3) a randomised, placebo-controlled trial. Lancet 2019; 394: 1713-23.

54 Whitesides LW, Baren JM, Biros MH, et al. Impact of individual clinical outcomes on trial participants' perspectives on enrollment in emergency research without consent. Clin Trials 2017; 14: 180-86.

55 Furyk J, McBain-Rigg K, Watt K, et al. Qualitative evaluation of a deferred consent process in paediatric emergency research: a PREDICT study. BMJ Open 2017; 7: e018562.

56 Shamy MCF, Dewar B, Chevrier S, et al. Deferral of consent in acute stroke trials. Stroke 2019; 50: 1017-20.

57 Harron K, Woolfall K, Dwan K, et al. Deferred consent for randomized controlled trials in emergency care settings. Pediatrics 2015; 136: e1316-22.

58 Honarmand K, Belley-Cote EP, Ulic D, et al. The deferred consent model in a prospective observational study evaluating myocardial injury in the intensive care unit. J Intensive Care Med 2018; 33: 475-80.

59 CONTRAST consortium. CONTRAST consortium. https://www. contrast-consortium.nl (accessed Jan 1, 2020).

60 Berkhemer OA, Fransen PSS, Beumer D, et al. A randomized trial of intraarterial treatment for acute ischemic stroke. $N$ Engl J Med 2015; 372: 11-20.

61 Jansen TC, Bakker J, Kompanje EJ. Inability to obtain deferred consent due to early death in emergency research: effect on validity of clinical trial results. Intensive Care Med 2010; 36: 1962-65.

62 United States Department of Health and Human Services. Food and Drug Administration. Guidance for institutional review boards, clinical investigators, and sponsors. Exception from informed consent requirements for emergency research. 2013. https://www. fda.gov/media/80554/download (accessed Dec 19, 2019).

63 Eltorki M, Uleryk E, Freedman SB. Waiver of informed consent in pediatric resuscitation research: a systematic review. Acad Emerg Med 2013; 20: 822-34.

64 Fehr AE, Pentz RD, Dickert NW. Learning from experience: a systematic review of community consultation acceptance data. Ann Emerg Med 2015; 65: 162-71.

65 Feldman WB, Hey SP, Franklin JM, Kesselheim AS. Public approval of exception from informed consent in emergency clinical trials: a systematic review of community consultation surveys. JAMA Netw Open 2019; 2: e197591. 
66 Eubank L, Lee KS, Seder DB, et al. Approaches to community consultation in exception from informed consent: analysis of scope, efficiency, and cost at two centers. Resuscitation 2018; 130: 81-87.

67 Harvin JA, Podbielski JM, Vincent LE, et al. Impact of social media on community consultation in exception from informed consent clinical trials. J Surg Res 2019; 234: 65-71.

68 Klein L, Moore J, Biros M. A 20-year review: the use of exception from informed consent and waiver of informed consent in emergency research. Acad Emerg Med 2018; 25: 1169-77.

69 Dickert NW, Scicluna VM, Baren JM, et al. Patients' perspectives of enrollment in research without consent: the patients' experiences in emergency research-progesterone for the treatment of traumatic brain injury study. Crit Care Med 2015; 43: 603-12.
70 Johnson LR, Siddaiah R. Use of deferred consent for enrolment in trials is fraught with problems. BMJ 2015; 351: h4609.

71 Goldstein CE, Weijer C, Brehaut JC, et al. Ethical issues in pragmatic randomized controlled trials: a review of the recent literature identifies gaps in ethical argumentation. BMC Med Ethics 2018; 19: 14

(C) 2020 Elsevier Ltd. All rights reserved. 